\title{
Gravitational waves at their own gravitational speed
}

\author{
C. S. Unnikrishnan ${ }^{1}$ and George T. Gillies ${ }^{2}$ \\ ${ }^{1}$ Gravitation Group, Tata Institute of Fundamental Research, \\ Homi Bhabha Road, Mumbai - 400 005, India \\ ${ }^{2}$ School of Engineering and Applied Science, \\ University of Virginia, Charlottesville, VA 22904-4746, USA \\ E-mail address: ${ }^{1}$ unni@tifr.res.in, ${ }^{2}$ gtg@virginia.edu
}

This essay received honorable mention in the 'Gravity Research Foundation 2018 Awards for Essays on Gravitation'.

\begin{abstract}
Gravitational waves propagate at the speed of light in general relativity, because of its special relativistic basis. However, light propagation is linked to the electromagnetic phenomena, with the permittivity and permeability constants as the determining factors. Is there a deeper reason why waves in a geometric theory of gravity propagate at a speed determined by electromagnetic constants? What is the relation between gravity's own constants and the speed of gravitational waves? Our attempt to answer these fundamental questions takes us far and deep into the universe.
\end{abstract}


The first guesses that light was linked to the phenomenon of electricity and magnetism were after Wilhelm Weber's formulation of electrodynamics in the 1850s that unified the experimental inputs from Coulomb and Ampère [1, 2]. Maxwell's theoretical triumph in finding the unified equations and their wave solution established that the speed of light was determined by the coupling constants of electricity and magnetism, $c^{2}=1 / \varepsilon_{0} \mu_{0}$. Therefore, the speed of light could be determined purely from measurements of forces in electrostatics and magnetostatics [3]. We want to pursue the similar natural question of whether or not we can determine the nature of propagation of gravitational waves entirely from the measured forces of gravity and gravitomagnetism, or from the forces of geometro-statics.

This is important conceptually and physically because there is no reason why waves in the physical or geometric theory of gravity propagate at a speed determined by the constants of another interaction, electromagnetism. Gravitational waves have some similarities and even more dissimilarities to electromagnetic waves. After all, one should have been able to build the fundamental theory of gravity without referring to the 'speed of light'. The historical priority to the electromagnetic reference is through the common relativistic basis. What is the relation between gravity's own constants and the speed of gravitational waves? Being the waves described in a geometric theory of gravity, with the Newtonian coupling constant $G$, their propagation should be determined entirely by gravitational coupling and, perhaps, some geometrical considerations. Conventionally, the theoretical reason is taken for granted, and quoted often, that because of the special relativistic basis of general relativity, the propagation of weak gravitational waves in linearized general relativity is at the speed of light. The physical surprise remains why the propagation of the waves of gravity are determined by electromagnetic constants. However, had we already known of the existence and propagation of gravitational waves at a time when the link between electromagnetism and light was established, before 1900, then surely we would have searched for a physical link between electromagnetism and gravity, and also a relation between ' $c$ ' and the gravitational coupling.

The electric permittivity of empty space, of the old ether, can be determined from the measurement of the magnitude of the force between known charges, using the Coulomb's law,

$$
F=k_{e} \frac{Q_{1} Q_{2}}{r^{2}}=\frac{1}{4 \pi \varepsilon_{0}} \frac{Q_{1} Q_{2}}{r^{2}}
$$


Similarly, the force between two 'magnetic poles' is

$$
F=k_{m} \frac{p_{1} p_{2}}{r^{2}}=\frac{\mu_{0}}{4 \pi} \frac{p_{1} p_{2}}{r^{2}}
$$

The magnetic permeability constant $\mu_{0}$ is measured from the force between current carrying conductors separated by $d$, using Ampère's law for the force between two currents,

$$
F=-\frac{\mu_{0} I_{1} I_{2}}{2 \pi d}
$$

After the realization that the ratio of the Coulomb constant and the Ampère constant - the ratio of the electric and magnetic force constants $-k_{e} /\left(\mu_{0} / 4 \pi\right)=1 / \varepsilon_{0} \mu_{0}$ is equal to the square of the velocity of light $c^{2}$, modern measurements are defined from just the value of ' $c$ ', with $\mu_{0}$ defined as $4 \pi \times 10^{-7} \mathrm{kgm} / C^{2}$. What are the relevant similar constants of gravity that determine the speed of gravitational waves and how do we measure them?

Well before the study of the electric force itself, the 'electric' aspect of gravity was studied extensively after the discovery of Newtonian gravitation, culminating in the measurement of the constant $G$ by Cavendish from the famous inverse square law

$$
F=-G \frac{m_{1} m_{2}}{r^{2}}
$$

But there was no 'magnetic gravity' seen in nature and nobody guessed or expected it, despite some theoretical clues, till general relativity predicted it. Gravitomagnetism, since then, has been well studied [4, 5, 6, 17, 8]. But direct measurements became possible only recently [9, 10].

We now explore the possibility of expressing the velocity of the gravitational waves, confirmed to be the numerical value ' $c$ ' from the joint LIGOFermi observation of the gravitational waves and light from the binary neutron stars merger [11, entirely in terms of the force constants from gravitational measurements of the 'electric' and 'magnetic' effects in gravity. This will free the physical existence of waves of the gravitational fields from the link to electrodynamic phenomena as well as the theoretical structure of relativity, Lorentz transformations etc. While all interactions are subject to dynamics allowed by relativity, each should be describable entirely in terms of its intrinsic constants, much like the situation with electromagnetic waves. The equivalent of permittivity in gravity is of course $1 / G$, measured in the Cavendish experiment, which was similar to the Coulomb experiment. We need a measurement of gravitomagnetism between known gravitomagnetic 
charges to get a value of the gravitational permeability $\mu_{G}$. Ideally, this would be an Ampère type experiment in which forces between currents of the charges of gravity, or mass currents, are measured. However, in the absence of that possibility, the next ideal measurement is of the gravitomagnetic interaction between spinning objects because spin, or angular momentum, is the closed current of the charge of gravity This is available from the direct measurement of the gravitomagnetic precession of the gyroscope in the Gravity Probe-B experiment 10, and from the analysis of the precession of orbits of the LAGEOS satellites [9]. We use the direct measurement of the gyroscope precession in the GP-B experiment. While these measurements have relatively large systematic errors, they are nevertheless the only direct measurements available and are quite suitable for our purpose. Since we know the precession rate and the 'gravitomagnetic moment' (i.e., the angular momentum) of the source, which is the Earth, we can estimate $\mu_{G}$. The gravitomagnetic interaction between the gyroscope with spin $s$ and the earth with angular momentum $J$ is

$$
E=\mu_{G} s \cdot J / R^{3}
$$

The precession of the gyroscope, averaged over an orbit assuming a circular polar orbit [10] is

$$
\langle\omega\rangle=\frac{\mu_{G} J}{2 R^{3}}=\frac{\mu_{G} I \Omega}{2 R^{3}} \simeq \frac{\mu_{G}(0.33) M R_{e}^{2} \Omega}{2 R^{3}}
$$

The normalized moment of inertia $I / M R^{2}=0.33$ is from the earth model [12] and $J \simeq 6 \times 10^{33} \mathrm{kgm}^{2} / \mathrm{s}$.

The Gravity Probe B orbit was at $R=R_{E}+640 \mathrm{~km}$. From the measured (average over 4 gyros) precession rate, $37.2 \pm 7.2$ milli-arcseconds/year, (5.7 \pm 1.1) $\times 10^{-15} \mathrm{radian} / \mathrm{s}$, we get

$$
\mu_{G} \simeq 6.7( \pm 1.3) \times 10^{-28} \mathrm{~m} / \mathrm{kg}
$$

We find, $\mu_{G} \varepsilon_{G}=\mu_{G} / G \simeq(1.0 \pm 0.2) \times 10^{-17} \mathrm{~s}^{2} / \mathrm{m}^{2}$. Thus, $\left(\mu_{G} / G\right)^{-1} \simeq$ $(9.9 \pm 1.9) \times 10^{16} \mathrm{~m}^{2} / \mathrm{s}^{2} \simeq c_{g}^{2}$.

This is a very satisfactory result. We have a description of the relativistic gravitational effects in the wave sector in terms of the purely gravitational constants $G$ and $\mu_{G}$, much like the description of electromagnetic effects in terms of $\varepsilon_{0}$ and $\mu_{0}$. We did not use the constant ' $c$ ' in our analysis, but verified 
that the product $G / \mu_{G} \simeq c_{g}^{2}$, the square of a velocity close to the empirically known velocity of gravitational waves. That it is also the velocity of light, of electromagnetism, reflects the universality of relativity. This exercise was similar to W. Weber's analysis in which he found that the product of the electric and magnetic force constants were related to the known velocity of light.

We have deeper physics linking gravity and electromagnetism, to be explored in the new relation,

$$
G / \mu_{G}=1 / \varepsilon_{0} \mu_{0}
$$

Since the values of ' $c$ ' and ' $\mu_{0}$ ' are exactly defined in the modern system, and with one equation, there is only one independent fundamental constant - $G$ - for the wave sector of the two long range interactions, gravity and electromagnetism. This shows the need and importance of measurements of $G$ at higher precision.

The inverse of the gravitational permeability with units $\mathrm{kg} / \mathrm{m}$ and its enormous value is suggestive of a 'massive compact' region. Though it is a familiar relation in the context of black holes, the correct identification, however, leads us to a surprise. Since $\mu_{G}$ is fundamental constant, it should be associated with the universe itself, and we have the relation

$$
1 / \mu_{G} \simeq 2 M_{u} / R \simeq 2 \frac{4 \pi}{3} \rho_{u} R^{2}=\frac{8 \pi}{3} \rho_{u} R^{2}
$$

where $\rho_{u}$ is the average density of the universe and $R$ is a length scale of the order of the Hubble radius. Thus the product of gravity's constants, $G$ and $1 / \mu_{G}$ is like the gravitational potential of the matter-energy of the entire universe, and it is numerically equal to $c_{g}^{2}$. This indicates the decisive role of the matter-energy content of the universe in relativistic physics [13].

We conclude that there is a self-contained description of the propagation of gravitational waves in terms of only the constants of gravity, without linking them to the electromagnetic constants and the speed of light. Gravitational waves propagate at a speed determined by its interaction constants, $G$ and $\mu_{G}$, entirely independently measurable in the laboratory and in space-based gravitation experiments. The two measurements we needed were the Cavendish measurement of $G$, the analog of $1 / \varepsilon_{0}$, and the direct measurement of the gravitomagnetic interaction between the spinning earth and a gyroscope, which gave us the gravitomagnetic permeability $\mu_{G}$. The 
link $\mu_{G} / G=\mu_{0} \varepsilon_{0}$ is through the nature's implementation of the principle of relativity. As not entirely unexpected, the matter-energy content in the universe plays a decisive role in gravitomagnetic effects, with a Machian flavor, and invites us for further exploration.

\section{References}

[1] W. E. Weber, Wilhelm Weber's Werke, Vol. 3: Galvanismus und Elektrodynamik, part 1 (Berlin: Julius Springer, 1893).

[2] A. K. T. Assis, Weber's Electrodynamics, Dordrecht: Kluwer Academic Publishers (1994).

[3] J. C. Maxwell, A Treatise on Electricity and Magnetism, New York: Dover (1954).

[4] A. Einstein, The Meaning of Relativity (Princeton University Press, 5th Edition 2014).

[5] W. de Sitter, On Einstein's Theory of Gravitation, and its Astronomical Consequences, MNRAS 77, 155 (1916).

[6] L. I. Schiff, Possible New Experimental Test of General Relativity Theory, Phys. Rev. Lett. 4, 215 (1960).

[7] K. S. Thorne, Gravitomagnetism, Jet in Quasars, and the Stanford Gyroscope Experiment, in Near Zero: New Frontiers of Physics (W. D. Fairbank, B. S. Deaver, Jr., C. W. F. Everitt and P. F. Michelson, Eds., W. H. Freeman \&Co., NY, 1988).

[8] I. Ciufolini and J. A. Wheeler, Gravitation and Inertia (Princeton University Press, Princeton, New Jersey, 1995).

[9] I. Ciufolini and E. C. Pavlis, A confirmation of the General Relativistic Prediction of the Lense-Thirring Effect, Nature 431, 958-960 (2004).

[10] C. W. F. Everitt et al., Gravity Probe B: Final Results of a Space Experiment to Test General Relativity, Phys. Rev. Lett. 106, 221101 (2011). 
[11] B. P. Abbot et al (LIGO Scientific collaboration and Virgo collaboration) GW17081\%: Observation of Gravitational Waves from a Binary Neutron Star Inspiral, Phys. Rev. Lett. 119, 161101 (2017).

[12] H. Jeffreys, The Earth (Cambridge University Press, London, 5th ed.1970).

[13] C. S. Unnikrishnan, Physics in the 'Once-Given' Universe, in S. Ghosh and G. Kar (Eds.), Recent Developments in Theoretical Physics, (World Scientific, Singapore, 2011), pp. 99-120. 